\title{
String Generating Functions and Spectral Functions of Hyperbolic Geometry
}

\section{E. X. Guimarães}

Instituto de Física, Universidade Federal Fluminense Av. Gal. Milton Tavares de Souza, s/n CEP 24210-346, Niterói-RJ, Brazil

E-mail: emilia@if.uff.br

\section{R. M. Luna}

Departamento de Física, Universidade Estadual de Londrina, Caixa Postal 6001,

Londrina-Paraná, Brazil

E-mail: luna@uel.br

\begin{abstract}
We discuss the homological aspects of a connection between quantum string generating function and formal power series associated with dimensions of chains and homologies of suitable Lie algebras. Our analysis can be considered as a new straightforward application of the machinery of modular forms and spectral functions (with values in the congruence subgroup of $S L(2, \mathbb{Z})$ ) to the topological vertex and the partition functions of Lagrangian branes, represented by means of formal power series that encode Lie algebra properties. The common feature in our examples lie in the modular properties of the characters of certain representations of the pertinent affine Lie algebras, and in the role of Selberg-type spectral functions of hyperbolic three-geometry associated with $q$-series in the calculation of string amplitudes.
\end{abstract}

Third International Satellite Conference on Mathematical Methods in Physics - ICMP 2013

21 - 26 October, 2013

Londrina - PR (Brazil) 


\section{Introduction}

We deal in this paper with applications of modular forms (and spectral functions related to the congruence subgroup of $S L(2, \mathbb{Z})$ ) to the topological vertex and string generating functions. For mathematicians topological vertex (and the respective string generating functions) may be associated to new mathematical invariants for spaces while, for physicists, they are link to quantum string partition functions.

Having given the general glance let us now explain the connection between the specific contents of the various sections in more detail. In Sect. 2 we will explore a remarkable connection between Poincaré polynomials (generating functions) and formal power series associated with dimensions of chains and homologies of Lie algebras (Euler-Poincaré formula). From a concrete point of view this paper consists on applications of spectral functions to quantum string partition functions connected to suitable Lie algebras.

We show that the Poincaré polynomials (Sect. 3) and the associated topological vertex and string amplitude can be converted into product expressions which inherit cohomological properties (in the sense of characteristic classes of foliations [1]) of appropriate polygraded Lie algebras.

The final result for a single Lagrangian brane and stack of branes (Sect. 4) is written in terms of spectral functions of the hyperbolic three-geometry associated with $q$-series.

\section{Graded algebras and spectral functions of hyperbolic geometry}

Before considering topological vertex and string amplitudes we would like to spend some time on the relation between formal power $q$-series and homologies of Lie algebras. We would like to show how combinatorial identities could be derived from initial complex of (graded) Lie algebras. Our interest is the Euler-Poincare formula associated with a complex consisting of linear spaces. The relations between Lie algebras and combinatorial identities was first discovered by Macdonald; the Euler-Poincaré formula is useful for combinatorial identities known as Macdonald identities. The Macdonald identities are related to Lie algebras in one way or another and can be associated with generating functions in quantum theory.

Let $\mathfrak{g}$ be an Lie algebra, and assume that it has a grading, i.e. $\mathfrak{g}$ is a direct sum of homogeneous components $\mathfrak{g}_{(\lambda)}$, where the $\lambda$ 's are elements of an abelian group, $\left[\mathfrak{g}_{(\lambda)}, \mathfrak{g}_{(\mu)}\right] \subset \mathfrak{g}_{(\lambda+\mu)}$. Let us consider a module $\mathbf{k}$ over $\mathfrak{g}$, or $\mathfrak{g}$-module, which is a left module over the universal enveloping algebra $U(\mathfrak{g})$ of $\mathfrak{g}$. Let $C^{n}(\mathfrak{g} ; \mathbf{k})$ be the space of $n$-dimensional cochain of the algebra $\mathfrak{g}$, with coefficients in $\mathbf{k}$. $d=d_{n}: C^{n}(\mathfrak{g} ; \mathbf{k}) \rightarrow C^{n+1}(\mathfrak{g} ; \mathbf{k})$ as $d_{n+1} \circ d_{n}=0$, for all $n$, the set $C^{\sharp}(\mathfrak{g} ; \mathbf{k}) \equiv$ $\left\{C^{n}(\mathfrak{g} ; \mathbf{k}), d_{n}\right\}$ is an algebraic complex, while the corresponding cohomology $H^{n}(\mathfrak{g} ; \mathbf{k})$ is referred to as the cohomology of the algebra $\mathfrak{g}$ with coefficients in $\mathbf{k}$.

Let $C_{n}(\mathfrak{g} ; \mathbf{k})$ be the space of $n$-dimensional chains of the Lie algebra $\mathfrak{g}$ and $\delta=\delta_{n}: C_{n}(\mathfrak{g} ; \mathbf{k}) \rightarrow$ $C_{n-1}(\mathfrak{g} ; \mathbf{k})$. The homology $H_{n}(\mathfrak{g} ; \mathbf{k})$ of the complex $\left\{C_{n}(\mathfrak{g} ; \mathbf{k}), \delta_{n}\right\}$ is referred to as the homology of the algebra $\mathfrak{g}$. We get $d\left(C_{(\lambda)}^{n}(\mathfrak{g} ; \mathbf{k})\right) \subset C_{(\lambda)}^{n+1}(\mathfrak{g} ; \mathbf{k})$ and $\delta\left(C_{n}^{(\lambda)}(\mathfrak{g} ; \mathbf{k})\right) \subset C_{n-1}^{(\lambda)}(\mathfrak{g} ; \mathbf{k})$ and both spaces acquire gradings. The chain complex $C_{\sharp}(\mathfrak{g}), \mathfrak{g}=\oplus_{\lambda=1}^{\infty} \mathfrak{g}_{(\lambda)}, \operatorname{dim} \mathfrak{g}_{(\lambda)}<\infty$, can be decomposed as

$$
0 \longleftarrow C_{0}^{(\lambda)}(\mathfrak{g}) \longleftarrow C_{1}^{(\lambda)}(\mathfrak{g}) \cdots \longleftarrow C_{N}^{(\lambda)}(\mathfrak{g}) \longleftarrow 0
$$


The well known Euler-Poincaré formula reads

$$
\sum_{m}(-1)^{m} \operatorname{dim} C_{m}^{(\lambda)}(\mathfrak{g})=\sum_{m}(-1)^{m} \operatorname{dim} H_{m}^{(\lambda)}(\mathfrak{g}) .
$$

As a consequence, we can introduce the $q$ variable and rewrite the identity (2.2) as a formal power series:

$$
\sum_{m, \lambda}(-1)^{m} q^{\lambda} \operatorname{dim} C_{m}^{(\lambda)}(\mathfrak{g})=\sum_{m, \lambda}(-1)^{m} q^{\lambda} \operatorname{dim} H_{m}^{(\lambda)}(\mathfrak{g})=\prod_{n}\left(1-q^{n}\right)^{\operatorname{dim} \mathfrak{g}_{n}} .
$$

In order to get the identity in its final form the homology $H_{m}^{(\lambda)}(\mathfrak{g})$ has to be computed.

Spectral generating functions for string amplitudes. Having given the general scheme let us now produce, for the benefit of the reader, the specific correspondence of the Poincare series (which can be associated with conformal structure in two dimensions [2]) and spectral functions of the three-dimensional hyperbolic geometry. Thus, the infinite product identities are [3, 1]:

$$
\begin{aligned}
\prod_{n=\ell}^{\infty}\left(1-q^{\mu n+\varepsilon}\right) & =\prod_{p=0,1} Z_{\Gamma}(\underbrace{(\mu \ell+\varepsilon)(1-i \rho(\tau))+1-\mu}_{s}+\mu(1+i \rho(\tau) p)^{(-1)^{p}} \\
& =\mathscr{R}(s=(\mu \ell+\varepsilon)(1-i \rho(\tau))+1-\mu), \\
\prod_{n=\ell}^{\infty}\left(1+q^{\mu n+\varepsilon}\right) & =\prod_{p=0,1} Z_{\Gamma}(\underbrace{(\mu \ell+\varepsilon)(1-i \rho(\tau))+1-\mu+i /(2 \operatorname{Im} \tau)}_{s}+\mu(1+i \rho(\tau) p))^{(-1)^{p}} \\
& =\mathscr{R}(s=(\mu \ell+\varepsilon)(1-i \rho(\tau))+1-\mu+i /(2 \operatorname{Im} \tau)),
\end{aligned}
$$

where $q=\exp (2 \pi i \tau), \rho(\tau)=\operatorname{Re} \tau / \operatorname{Im} \tau, \mu \in \mathbb{R}, \ell \in \mathbb{Z}_{+}$and $\varepsilon \in \mathbb{C}$. The Ruelle function $\mathscr{R}(s)$ is an alternating product of more complicate factors, each of which is a Selberg-type spectral function $Z_{\Gamma}(s)$ (the analytic and modular properties of the Patterson-Selberg spectral function $Z_{\Gamma}(s)$ the reader can find in [1]).

At this point one can use the Ruelle functions $\mathscr{R}(s)$ to naturally generalize the result (2.4), (2.5) for more general infinite product identities

$$
\begin{aligned}
\prod_{n=m}^{\infty}\left(1-q^{\mu n+\varepsilon}\right)^{v n} & =\mathscr{R}(s=(\mu m+\varepsilon)(1-i \rho(\tau))+1-\mu)^{v m} \\
& \times \prod_{n=m+1}^{\infty} \mathscr{R}(s=(\mu n+\varepsilon)(1-i \rho(\tau))+1-\mu)^{v}, \\
\prod_{n=m}^{\infty}\left(1+q^{\mu n+\varepsilon}\right)^{v n} & =\mathscr{R}(s=(\mu m+\varepsilon)(1-i \rho(\tau))+1-\mu+i /(2 \operatorname{Im} \tau))^{v m} \\
& \times \prod_{n=m+1}^{\infty} \mathscr{R}(s=(\mu n+\varepsilon)(1-i \rho(\tau))+1-\mu+i /(2 \operatorname{Im} \tau))^{v} .
\end{aligned}
$$

\section{Polygraded algebras and polynomial invariants}

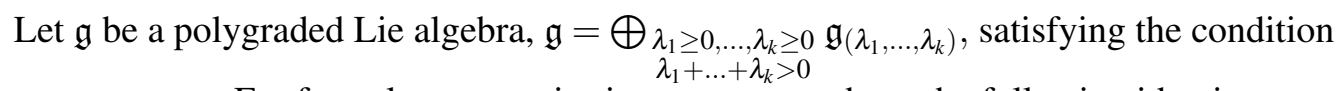
$\operatorname{dim} \mathfrak{g}_{\left(\lambda_{1}, \ldots, \lambda_{k}\right)}<\infty$. For formal power series in $q_{1}, \ldots, q_{k}$, we have the following identity:

$$
\sum_{m, \lambda_{1}, \ldots, \lambda_{k}}(-1)^{m} q_{1}^{\lambda_{1}} \cdots q_{k}^{\lambda_{k}} H_{m}^{\left(\lambda_{1}, \ldots, \lambda_{k}\right)}=\prod_{n_{1}, \ldots, n_{k}}\left(1-q_{1}^{n_{1}} \cdots q_{k}^{n_{k}}\right)^{\operatorname{dimg}_{n_{1}, \ldots, n_{k}}}
$$


We would like to stress that partition functions can indeed be converted into product expressions. The expression on the right-hand side of (3.1) looks like counting the states in the Hilbert space of a second quantized theory.

For more examples let us proceed to describing the properties of link homologies suggested by the their relation to Hilbert spaces of BPS states [4]. Let $\mathscr{H}_{k, j}^{\mathfrak{s l}(N) ; R_{1}, \ldots, R_{\ell}}(L)$ be the doubly-graded homology theory whose graded Euler characteristic is the polynomial invariant $\bar{P}_{\mathfrak{s l}(N) ; R_{1}, \ldots, R_{\ell}}(q)$ (the bar means that this invariant is unnormalized invariant; its normalized version obtained by dividing by the invariant of the unknot)

$$
\bar{P}_{\mathfrak{s l}(N) ; R_{1}, \ldots, R_{\ell}}(q)=\sum_{k, j \in \mathbb{Z}}(-1)^{j} q^{k} \operatorname{dim} \mathscr{H}_{k, j}^{\mathfrak{s l}(N) ; R_{1}, \ldots, R_{\ell}}(L) .
$$

Here $L$ is an oriented link in $S^{3}$, we consider the Lie algebra $\mathfrak{g}=\mathfrak{s l}(N)$ (there is a natural generalization to other classical Lie algebras B, C, and D [4]) and a link colored is given by a collection of representations $R_{1}, \ldots, R_{\ell}$ of $\mathfrak{s l}(N)$. In order to be agree with the standard notations we use the variables $\{q, t\},[5]$, when we discuss link homologies.

The graded Poincaré polynomial has the form [4]

$$
\bar{P}_{\mathfrak{s l}(N) ; R_{1}, \ldots, R_{\ell}}(q, t):=\sum_{k, j \in \mathbb{Z}} q^{k} t^{j} \operatorname{dim} \mathscr{H}_{k, j}^{\mathfrak{s l}(N) ; R_{1}, \ldots, R_{\ell}}(L) .
$$

By definition, it is a polynomial in $q^{ \pm 1}$ and $t^{ \pm 1}$ with integer non-negative coefficients. In addition, evaluating (3.3) at $t=-1$ gives (3.2). In the case $R_{a}=\square$ for all $a=1, \ldots, \ell$, the homology $\mathscr{H}_{k, j}^{\mathrm{sl}(N) ; R_{1}, \ldots, R_{\ell}}(L)$ is known as the Khovanov-Rozansky homology, ${ }_{(K R)} \bar{H}_{k, j}^{N}(L)$, and its graded Poincaré polynomial takes the form

$$
\overline{\mathscr{P}}_{\mathfrak{s l}(N) ; \square, \cdots, \square}(q, t)=\sum_{k, j \in \mathbb{Z}} q^{k} t^{j} \operatorname{dim}_{(K R)} \bar{H}_{k, j}^{N}(L) .
$$

The further physical interpretation of homological link invariants via Hilbert spaces of BPS states leads to certain predictions regarding the behavior of link homologies with rank $N$ (for more discussion see $[4,6])$.

\section{A single Lagrangian brane}

Let us recall the connection between topological vertex and open string amplitudes in the presence of stack of branes. It is known that in the case of a stack Lagrangian D-branes is ending on one of the legs of the $\mathbb{C}^{3}$; the partition function is given by

$$
\mathscr{F}(q ; V)=\sum_{v} C_{\emptyset \emptyset v}\left(q^{-1}\right) \operatorname{Tr}_{v} V .
$$

Here $\operatorname{Tr}_{v} V=s_{v}(\mathbf{x})$ are the Schur functions, $\mathbf{x}=\left\{x_{1}, x_{2}, \cdots\right\}$ are the eigenvalues of the holonomy matrix $V$, and $C_{\lambda \mu v}(q)$ is the topological vertex [7]. Recall that Schur functions have the property that $s_{v / \lambda}(Q)=Q^{|v|-|\lambda|}, v \succ \lambda$, and $s_{v / \lambda}(Q)=0$, otherwise.

For a single Lagrangian brane $\mathbf{x}=(-Q, 0,0,0, \cdots)$ we get the well known partition function

$$
\mathscr{F}(q ; Q)=\prod_{n=1}^{\infty}\left(1-Q q^{-n+\frac{1}{2}}\right) \stackrel{\text { by Eq. (2.4) }}{=} \mathscr{R}(s=(\alpha-1 / 2)(1-i \rho(\tau))+2) .
$$


In order to be careful and to agree with the standard notations, we use the variables $\{Q, q, t\}$ when we talk about topological string amplitudes computed by the topological vertex, cf. [8]. This set of variables are related with our notations $\left\{Q^{\alpha}, q=\exp (2 \pi i \tau), t=\exp (2 \pi i \sigma)\right\}$ as follows: $\tau=F_{1} / 2 \pi i, \sigma=F_{2} / F_{1}$, where we turn on a constant not self-dual graviphoton field strength $F=F_{1} d x^{1} \wedge d x^{2}+F_{2} d x^{3} \wedge d x^{4}$, and $\alpha=-\int_{C} \omega / F_{1}$ [8]. The above partition function of a single Lagrangian brane can be interpreted in terms of the Hilbert series of the the symmetric product of $\mathbb{C}$. Indeed, $s_{v}(Q)$ is non-zero only for those partitions for which $\ell(v)=1$. These are exactly the partitions which label the fixed points of the symmetric product of $\mathbb{C}$, i.e., $\operatorname{Sym} \bullet(\mathbb{C})$ has a single fixed point labelled by the partition $v=(\bullet, 0,0, \cdots)$. A generating function of the Hilbert series of the symmetric product is [9]

$$
\mathfrak{G}(\psi, q):=\sum_{k=0}^{\infty} \psi^{k} H\left[\operatorname{Sym}^{k}(\mathbb{C})\right](q) .
$$

In order to determine $H\left[\operatorname{Sym}^{k}(\mathbb{C})\right](q)$ note that the $R_{k}$ is the ring of symmetric functions in the variables $\left\{z_{1}, z_{2}, \cdots, z_{k}\right\}$ and therefore the Schur functions provide a basis of $R_{k}, R_{k}=\left\langle s_{V}\left(z_{1}, \cdots, z_{k}\right)\right|$ $\ell(v) \leq k\rangle$. The condition $\ell(v) \leq k$ is necessary since $s_{v}\left(z_{1}, \cdots, z_{k}\right)=0$ for $\ell(v)>k$. $R_{k}$ is isomorphic to the Hilbert space $\mathscr{H}_{k}$, generated by bosonic oscillator up to charge $k$. The Hilbert spaces $\left\{\mathscr{H}_{k}\right\}_{k=0}^{\infty}$ form a nested sequence $\mathscr{H}_{0} \subset \mathscr{H}_{1} \subset \mathscr{H}_{2} \subset \mathscr{H}_{3} \subset \cdots$ which corresponds to the nested sequence of Young diagrams of increasing number of rows.

The $\mathbb{C}^{\times}$action (on $\mathbb{C} q$ acts as a $\mathbb{C}^{\times}$action $z \mapsto q z$ ), which lifts to an action on the $\operatorname{Sym}(\mathbb{C})$ such that the Schur functions $s_{v}\left(z_{1}, \cdots, z_{k}\right)$ are eigenfunctions with eigenvalue $q^{|v|}$, becomes the action of $q^{L_{0}}$ on the states in $\mathscr{H}\left(L_{0}=\sum_{n>0} \alpha_{-n} \alpha_{n}\right)$,

$$
\begin{aligned}
H\left[R_{k}\right](q) & =\operatorname{Tr}_{\mathscr{H}_{k}} q^{L_{0}}=\sum_{v \mid \ell(v) \leq k} q^{|v|}=\prod_{n=1}^{k}\left(1-q^{n}\right)^{-1}=s_{(k)}\left(1, q, q^{2}, \cdots\right) \\
& =\left[\frac{\mathscr{R}(s=1-i \rho(\tau))}{\mathscr{R}(s=(k+1)(1-i \rho(\tau)))}\right]^{-1} .
\end{aligned}
$$

Note that the Hilbert series of $R_{k}$ in this case turns out to be the generating function of the number of partitions with at most $k$ parts. Thus the generating functions $\mathfrak{G}(\psi, q)$ is then given by

$$
\begin{aligned}
\mathfrak{G}(\psi, q) & =\sum_{k=0}^{\infty} \psi^{k} H\left[R_{k}\right](q)=\sum_{k=0}^{\infty} \psi^{k} \operatorname{Tr}_{\mathscr{H}_{k}} q^{L_{0}}=\sum_{k=0}^{\infty} \psi^{k} s_{(k)}\left(1, q, q^{2}, \cdots\right) \\
& =\sum_{k=0}^{\infty} s_{(k)}(\psi) s_{(k)}\left(1, q, q^{2}, \cdots\right)=\sum_{V} s_{V}(\psi) s_{V}\left(1, q, q^{2}, \cdots\right)=\sum_{V} s_{V}\left(q^{-\rho}\right) s_{V}\left(\psi q^{-\frac{1}{2}}\right) \\
& =\sum_{V} s_{v^{t}}\left(q^{\rho}\right) s_{V}\left(-q^{-\frac{1}{2}} \psi\right)=\sum_{V} C_{\emptyset \emptyset v}\left(q^{-1}\right) \operatorname{Tr}_{v} V=\mathscr{F}(q ; Q),
\end{aligned}
$$

where $\operatorname{Tr}_{v} V=s_{V}(Q)$ and $Q=q^{-\frac{1}{2}} \psi . \operatorname{Tr}_{v} V=s_{v}(\mathbf{x})$ where $\mathbf{x}=\left\{x_{1}, x_{2}, \cdots\right\}$ are the eigenvalues of the holonomy matrix $V$. Thus the partition function takes the form

$$
\begin{gathered}
\mathscr{F}(q ; V)=\sum_{V} C_{\emptyset \emptyset v}\left(q^{-1}\right) s_{V}(\mathbf{x})=\sum_{v} s_{V^{t}}\left(q^{\rho}\right) s_{V}(\mathbf{x})=\prod_{k, j=1}^{\infty}\left(1+q^{-k+\frac{1}{2}} x_{j}\right) \\
\underline{\underline{\text { by Eq. }(2.5)}} \prod_{j=1}^{\infty} \mathscr{R}\left(s=\left(a_{j}+1 / 2\right)(1-i \rho(\tau))+2-i /(2 \operatorname{Im} \tau)\right),
\end{gathered}
$$


where $a_{j} \equiv \log x_{j} / \log q$. If we move the brane to infinity $\left(Q=e^{-\int_{C} \omega} \mapsto 0\right)$ the contribution of the higher modes is suppressed. On the other hand as the brane moves towards the origin $(Q \mapsto 1)$ higher oscillator modes starts contributing with equal weight to the partition function. It is also follows that the topological vertex $C_{\emptyset \emptyset(k)}(q)$ has an interpretation as counting the number of states of a given energy in the Hilbert space $\mathscr{H}_{k}$. It is tempting to conjecture that the topological vertex with all three partitions non-trivial has a similar interpretation in terms of spectral functions.

Stack of Branes. Let us consider the case of multiple Lagrangian branes on the one of legs of $\mathbb{C}^{3}$. Then $\mathbf{x}=\left\{x_{1}, x_{2}, \cdots, x_{N}\right\}$ and the partition function becomes

$$
\begin{aligned}
\mathscr{F}(\mathbf{x}, q) & =\sum_{v} C_{\emptyset \emptyset v}\left(q^{-1}\right) s_{v}(\mathbf{x})=\prod_{j=1}^{N} \prod_{k=1}^{\infty}\left(1+q^{-k+\frac{1}{2}} x_{j}\right) \\
& =\prod_{j=1}^{N} \mathscr{R}\left(s=\left(a_{j}+1 / 2\right)(1-i \rho(\tau))+2-i /(2 \operatorname{Im} \tau)\right) .
\end{aligned}
$$

It is clear that the partition function (4.7) is the generating function of the Hilbert series of symmetric products of $\mathbb{C}$.

\section{Acknowledgements}

The authors would like to acknowledge CAPES (Brazil) and Fundação Araucária (Paraná, Brazil) for support.

\section{References}

[1] A. A. Bytsenko, M. Chaichian, R. J. Szabo and A. Tureanu, Quantum Black Holes, Elliptic Genera and Spectral Partition Functions, to be published in IJGMMP (2014); [arXiv:hep-th/1308.2177].

[2] A. A. Bytsenko and A. Tureanu, Quantum Corrections to Bekenstein-Hawking Black Hole Entropy and Gravity Partition Functions, Nucl. Phys. B 873 (2013) 534-549;[arXiv:hep-th/1304.7021].

[3] A. A. Bytsenko and M. E. X. Guimarães, Truncated Heat Kernel and One-Loop Determinants for the BTZ Geometry, Eur. Phys. J. C 58 (2008) 511-516; [arXiv:hep-th/0809.1416].

[4] S. Gukov, A. Iqbal, C. Kozçaz and C. Vafa, Link Homologies and the Refined Topological Vertex, Commun. Math. Phys. 298 (2010) 757-785; [arXiv:hep-th/0705.1368v1].

[5] N. Dunfield, S. Gukov and J. Rasmussen, The Superpolynomial for Knot Homologies, Experiment. Math. 15 (2006) 129-159; [arXiv:math.GT/05405662].

[6] S. Gukov, A. Schwarz and C. Vafa, Khovanov-Rozansky homology and topological strings, Lett. Math. Phys. 74 (2005) 53-74; [arXiv:hep-th/0412243].

[7] M. Aganagic, A. Klemm, M. Marino and C. Vafa, The Topological Vertex, Commun. Math. Phys. 254 (2005) 425-478; [arXiv:hep-th/0305132].

[8] A. Iqbal, C. Kozçaz and C. Vafa, The Refined Topological Vertex, JHEP 0910 (2009) 069 (70pp.); [arXiv:hep-th/0701156v2].

[9] D. Martelli, J. Sparks and S. T. Yau, The Geometric Dual of a-maximisation for Toric Sasaki-Einstein Manifolds, Commun. Math. Phys. 280 (2006) 39-65; [arXiv:hep-th/0503183]. 